\title{
Les pneumopathies du porc à Madagascar *
}

\author{
par J. J. RIBOT avec lo collaboration technique de M. RAZAFINDRATSITA Roger.
}

\begin{abstract}
RÉSUMÉ
On observe à Madagascar sur des porcelets de 2 à 5 mois des pneumopathies enzoołiques. La maladie sévit en saison fraîche, elle est favorisée par l'humidité de la porcherie, une alimentation déséquilibrée ef le parasitisme.

Ces pneumopathies évolvent de façon subaiguë ou chronique ef la terminaison mortelle est fréquente.

Sur 109 porcs autopsiés ef examinés au Laboratoire, les germes les plus fréquemment isolés ont été : Escherichio coli, Pasteurella multocido, Klebsiello pneumonioe, Pseudomonas aeruginoso.

Les moyens de lutfe acluels font appel à la prophylaxie sanitaire basée sur l'isolement des malades, l'amélioration des conditions d'hygiène du logement et d'alimentation et sur le déparasitage régulier. La prophylaxie médicale basée sur l'emploi de vaccins antipasteurellique et anti-pyocyanique a permis d'enrayer les enzooties dans certains élevages.
\end{abstract}

Lors d'autopsies pratiquées au service de diagnostics du laboratoire, des atteintes pulmonaires plus ou moins graves ont été fréquemment observées sur des porcs en général âgés de 2 à 5 mois (13). Ces pneumopathies accompagnent le plus souvent le parasitisme ef la malnutrition. Dans quelques cas néanmoins elles sont la complication d'autres maladies.

Systématiquement, la recherche des germes en cause a été effectuée et a donné les résultats qui sont exposés plus loin.

\section{1. — ÉPIDÉMIOLOGIE}

Les lésions pulmonaires sont trouvées toute l'année avec une fréquence plus marquée en juillet-août (mois froids), en janvier (mois chaud mais très humide) et en mai (début des froids nocturnes avec parasitisme important).

Les porcelets de 2 à 5 mois qui sont atteints, présentent des lésions pulmonaires que nous

* Communication au $18^{\circ}$ congrès mondial vétérinaire. Paris, 17-22 juillet 1967 . qualifierons de primitives. Chez les porcs adultes, les lésions du poumon accompagnent soit la maladie de Teschen, soit surtout une hépatitecirrhose (23).

\section{II. — LES LÉSIONS OBSERVÉES}

Elles vont de la simple congestion pulmonaire à la pleuro-pneumonie, purulente ou non, en passant par de nombreux stades intermédiaires tels que œdème pulmonaire, broncho-pneumonie, abcès pulmonaire (10-20-22).

Souvent et cela dans une zone délimitée des Hauts-Plateaux (Antsiriribe) une infestation massive par métastrongles accompagne la pneumonie.

\section{III. - LA MALADIE PRIMITIVE}

Nous désignons ainsi une pneumopathie désignée classiquement par Von GOIDSENHOVEN et SCHOENAERS (31) ef par DUNNE (4) sous les termes de broncho-pneumonie des porcelets, pasteurellose porcine, pneumo-entérite du porc, pyobacillose porcine. 
La maladie, d'allure enzootique, et d'évolution subalguë ou chronique se termine fréquemment par la mort. Toux, jetage, difficulté respiratoire, dyspnée, matité, râles à l'auscultation constituent les symptômes caractéristiques.

Souvent la maladie s'accompagne de troubles digestifs (entérite diarrhéique) qui fait maigrir les animaux. De nombreux facteurs débilitants, en premier lieu le parasitisme favorisent ces pneumopathies.

La recherche systématique de métastrongles n'a pas toujours été falte sur les 109 cadavres autopsiés.

Néanmoins, 14 porcelets étaient atteints de façon certaine de strongylose pulmonaire. Notre opinion rejoint celle de MACKENZIE (19) qui a démontré le rôle néfaste et aggravant des métastrongles pulmonarres.

A côté du parasitisme «spécifique» du poumon, les parasites intestınaux diminuent les défenses de l'organisme infesté (6-7). Certains de ces parasites (ascarides, strongiloïdes) du fait de leur cycle parasitaire, passant par le poumon, contribuent pour une large part à favoriser l'éclosion des pneumopathies et suivant l'expression de Stefanski (28) « ouvrent la porte aux microbes $»$ qu'ils transportent.

La recherche des parasites intestinaux n'a pu être faite systématiquement sur les 109 cadavres. Nous avons cependant relevé dans nos régistres d'autopsies l'infestation de 28 de ces porcs pas Ascarides (30) et Acanthocéphales.

A côté de ces parasites, on retrouve fréquemment trichures, arduenna, œsophagostomes.

En outre, malnutrition (3) avitaminoses (16) sont d'autres facteurs d'éclosion de maladies infectieuses habituellement rencontrés en milieu tropical.

\section{IV. - LA PNEUMOPATHIE SECONDAIRE}

Dans ce cas la lésion pulmonaire fait suite à une autre maladie. Les porcs atteints sont plus âgés, le plus souvent, il s'agit d'adultes.

La maladie que l'on doit incriminer en premier lieu à Madagascar est l'hépatite-cirrhose due à la présence d'aflatoxine dans certains aliments (tourteaux d'arachides) entrant dans la ration $(23,25)$.

Treize fois, sur soixante-huit cas d'hépatite- cirrhose relevés dans notre service, nous avons trouvé conjointement sur les cadavres des lésions de cirrhose ef de pneumonie.

En deuxième lieu, l'atteinte pulmonaire microbienne peut être une complication de maladie virale. A Madagascar, c'est presque uniquement la maladie de Teschen (26) qui est en cause et qui a été diagnostiquée dans onze cas.

La peste porcine européenne existe depuis peu à Madagascar, sa diffusion a pu être limitée. Sur un seul cadavre autopsié, la pneumonie pouvait être considérée comme une complication de la peste.

\section{V. - LES GERMES RENCONTRÉS}

Des germes très divers, qu'il s'agisse de pneumopathie primitive ou secondaire ont été isolés. A côté de germes que l'on peut qualifier de spécifiques, très souvent, des germes entériques, sans spécificité pulmonaire, ont été identıfiés.

Le tableau mérite quelques commentaires.

a) Escherichia Coli : est le germe le plus fréquemment isolé, 43 cas (24). Sur ces 43 cas, 23 fois le colibacille a été isolé seul ou associé à des germes entériques (Hafnio, Alcoligenes, Protéus Providencia, Citrobacter, Bacillus).

Sur ces 23 cas, il s'agissait, outre les lésions pulmonares de :

(9),

- 10 porcs présentant une hépatite-cirrhose

- 6 porcs présentant la maladie de Teschen,

- 6 porcs présentant une gastro-entérite,

- 1 porc sans lésion intestinale visible, mais parasité par Ascaris (30).

Dans les 20 autres cas, le colibacille était associé à des germes nettement plus pathogènes que lui et plus spécifiquement pulmonaires (Pasteurella multocida, Klebsiella pneumoniae, Streptococcus hémolyticus).

b) Pasteurella multocida : est très souvent le germe causal.

La pasteurella a été isolée 25 fois dont 16 en culture pure (15).

Sur 9 prélèvements, on l'a trouvé associée à :

Klebsiello pneumoniae (4 fois).

Pseudomonas aeruginosa ( 3 fois). 
TABLEAU

\begin{tabular}{|c|c|c|c|}
\hline Ge $\mathrm{r}$ me $\mathrm{s}$ & Culture pure & Culture mélangée & Total \\
\hline Escherichia Coli & 23 & 20 & 43 \\
\hline Pasteurelza Multocida & 16 & 9 & 25 \\
\hline Klebsiella Pneumoniae & 4 & 19 & 23 \\
\hline Pseudomonas Aemuginosa & 2 & 7 & 9 \\
\hline Streptocoque hémolytique & 1 & 6 & 7 \\
\hline Cormebacterium Pyogenes & - & 3 & 3 \\
\hline Bordetelia Bronchiseptica & - & 2 & 2 \\
\hline Staphy tocoques pathogènes & - & 1 & 1 \\
\hline Salmonetza typhymiritom & - & 2 & 2 \\
\hline Salmonetza cholerae suis & - & 1 & 1 \\
\hline & 46 & 70 & 116 \\
\hline
\end{tabular}

Escherichio Coli (2 fois).

Streptococcus hémolyticus (1 fois).

c) Klebsiella pneumoniae : arrive en 3 e position car isolée 23 fois dont 4 seulement en culture pure.

Les germes le plus souvent associés sont par ordre d'importance : $E$. Coli (7 fois) Posteurella (5 fois) Pseudomonos (3 fois) Bordefella (1 fois) puis Streptococcus, Alcaligènes, Hafnia, Protéus (1 fois).

d) Pseudomonos aeruginosa : Le bacille pyocyanique (2) coloré ou variété achromogène a été isolé sur 9 poumons. Deux fois isolé pur, 3 fois associé à Posteurella, 3 fois à Klebsiella, une fois à Protéus ce germe a un rôle indéniable dans les pneumopathies primitives des porcelets.

e) Autres germes rencontrés : Nous avons également isolé des poumons lésés :

Streptococcus hémolytıcus ( 7 fois).

Corynebocterium pyogenes (3 fois).

Bordetella Bronchisepfica (2 cas) (5-8).

Stophylococcus aureus (1 cas).

En outre, il a été possible d'isoler de la moelle osseuse des porcs présentant des lésions pulmonaires :

Solmonella typhi-murium : 2 cas.

Salmonella Choleroe suis : 1 cas.

Il s'agissait pour Salmonella cholerae suis d'un porc atteint par ailleurs de peste porcine euro- péenne et pour Salmonella typhimurium de deux porcelets originaires de la même région, atteints de pneumonie vermineuse à métastrongles et parasités en outre par des Ascarides, des Trichures et des Fsophagostomes.

Les bactéries les plus spécifiques sont donc Pasteurella multocida, Klebsiella pneumoniae et Pseudomonas aeruginosa. Ce sont des germes classiques qui sont retrouvés par grand nombre d'auteurs dons des cas similaires.

\section{VI. - LES MOYENS DE LUTTE}

S'il est exact en Europe que « les maladies du porc ont changé de visage » (18) cela se vérifie beaucoup moins en milieu tropical; milieu plus neuf mais aussi moins exploré.

Cependant, les «maladies de la civilisation» guettent à brève échéance les pays tropicaux qui tendent à orienter leurs élevages vers le mode industriel ou semi-industriel afin d'en augmenter la productivité. L'hépatite-cirrhose due à l'aflatoxine des tourteaux d'arachides en est un exemple.

Néanmoins, encore actuellement, les moyens de lutte contre les maladies en général, doivent faire appel essentiellement (et c'est le cas des pneumopathies des porcs) à la prophylaxie sanitaire.

Le service vétérinaire lutte pour améliorer au maximum les conditions d'hygiène, du logement 
et de l'alimentation (16). Du point de vue logement, la porcherie à sol cimenté en pente douce, bien exposée et aérée (12) telle qu'elle est classiquement conçue (11) est un idéal qui se heurte souvent à Madagascar à la peur du vol. En effet par crainte d'être volés, les propriétaires confinent leurs animaux dans des locaux ne comportant qu'une porte, d'ailleurs solidement cadenassée. De ce fait les désinfections préconisées n'ont pas toujours les bons résultats escomptés (14-17).

Du point de vue hygiène alimentaire, l'utilisation de concentrés semble devoir être une arme efficace (27) contre les carences.

Une législation est à l'étude pour exclure systématiquement de l'alimentation animale les tourtecux contenant de l'aflatoxine. Ce rejet des tourteaux toxiques évitera les dystrophies hépatiques et par tant certaines pneumopathies secondaires (29-21).

Enfin le déparasitage systématique est vulgarisé par les agents du service de l'élevage.

Malheureusement, l'éloignement, la dispersion des élevages et le manque de personnel ne permettent que d'atteindre une faible partie de la population, donc des animaux.

La prophylaxie médicale, basée sur l'emploi d'un vaccin antipasteurellique et antipyocyanique a permis d'enrayer les enzooties observées dans des élevages assez importants (50 porcs et plus) donc mieux surveillés.

Ce vaccin est préparé à partir d'une culture de Pasteurella multocida tuée par le formol $(0,5$ p. 100) et d'une culture de Pseudomonas aeruginosa tuée par la chaleur $\left(1\right.$ heure à $\left.70^{\circ} \mathrm{C}\right)$.

Après mélange des cultures, un volume égal de gel d'alumine est ajouté comme adjuvant.
Ce vaccin employé à la dose de $5 \mathrm{~cm}^{3}$ par voie sous-cutanée a danné des résultats encourageants et nous en préparons plusieurs milliers de doses annuelles.

II ne peut être question d'employer des médications variées en particulier les antibiotiques dont le prix de revient est trop élevé (32), pour des élevages dont la rentabilité est par trop soumise aux fluctuations des marchés et des cours des produits. Le vaccin préparé revient aux environs de 7 FMG la dose alors que le porc se vend sur la base de 150 FMG le kilo vif.

Les pneumopathies du porc, ef c'est essentiellement à la maladie primitive que nous pensons, ne sont pas actuellement l'obstacle essentiel à l'élevage porcin malgache. Elles méritent cependant d'être prises très sérieusement en considération afin d'en éviter la diffusion. La meilleure prophylaxie consiste pour le moment, à notre avis, à vulgariser dans tout le pays la pratique de l'élevage des porcs sur sol cimenté, le déparasitage interne systématique et l'emploi de concentrés alimentaires permettant d'équilibrer des rations qui sont exclusivement composées d'un des trois produits suivants : manioc, mais ou son de riz.

Ces mesures simples, l'expérience l'a démontré, permettent à des animaux sains, nourris correctement, de réaliser outre-mer dans certaines zones (Hauts-Plateaux malgaches par exemple) des performances égales à celles des porcins d'Europe.

\section{SUMMARY}

\section{Pulmonary diseases of pig in Madagascar}

Enzootic pulmonary diseases have been recorded in Madagascar in piglets from 2 to 5 months. Humidity of the housing, unbalanced diet and parasitic diseases are favourable factors of the disease, which occurs during the cool season.

The pulmonary diseases occur subacutely or chronically and the issue is very often fatal.

The germs, which have been mostly isolated in 109 pigs, after post-morten and laboratory examination, are : Escherichio coll, Pasteurello multocida, Klebsiella pneumoniae, Pseudomonas aeruginoso. 
The control of the disease is based on the isolation of infected animals, the improvement of the hygiene of housing and food, and a regular parasites conirol. The medical prophylaxis includes vaccination against Pasfeurella and B. pyocyoneus; Good results have been reported in some areas.

\section{RESUMEN}

Las neumopatias del cerdo en Madagascar

Se observan en Madagascar neumopatias enzooticas en cerditos de 2 a 5 meses de edad. La enfermedad ocurre durante la estación fresca. La humedad de la porqueriza, una alimentación desequilibrada y el parasitismo la favorecen.

Estas neumapatias evolucionan de modo subagudo o crónico y a menudo la muerte es frecuente. Se hicieron la autopsia y el examen en el laboratorio de 109 cerdos. Los germenes más frecuentemente aislados fueron : Escherichia coli, Pasteurella multocida, Klebsiella pneumoniae, Pseudomonos aeruginasa. La profilaxio sanitaria, incluyente el aislamiento de los enfermos, la mejoración de las condiciones de higiene del alojamienta y de la alımentación, y la utilización regular de parasiticidas, representa los medios actuales de lucha. La profilaxia medical con vacunas contra la pasteurelosis y la piocianosis permitió enrayar las enzoolıas en cierlas ganaderias.

\section{BIBLIOGRAPHIE}

1. BUGARD (P.), JOUBERT (L.) et HENRY (M.). - Maladies de civilisation ef dirigisme biologique. Masson et Cie, Editeurs, Paris.

2. BUTTIAUX (R.) et GAGNON (P.). Au sujet de la classification des Pseudomonas ef des Achromobacter. Annales 1. P., Lille, 1958, 59, $10: 121-149$.

3. DREUX (G.). - L'utilisation des produits de la ferme dans l'Elevage des porcs. Les maladles d'origine alimentaire. Leurs remèdes. Editions S. E. P., Paris (1).

4. DUNNE $(H . W$.$) . - Les maladies du porc.$ Vigot Frères, Editeurs, Paris.

5. DUNNE (H. W.), KRADEL (D. C.), DOTY (R. B.). - Bordetella bronchiseptica in pneumonia in young pigs. J. Amer. vet. Med. Ass., 1961, $139: 897-899$.

6. EUZEBY (J.). - Les maladies vermineuses des animaux domestiques. Tome I. Fasc. 1er. Vigot Frères, Editeurs, Paris (1961).

7. EUZEBY (J.) et RENAULT (L.). - Aspects du parasitisme helminthique chez le porc en France. Rev. Méd. Vét., 1966, 117, 12 : 1037-1057.

8. FARKAS-HIMSBY $\left(\mathrm{H}_{\text {. }}\right)$, - Differenciation of Pasteurella pseudotuberculosis and Bordefella bronchiseptica by simple biochemical tests. Amer. J. Vet. Res., 1963, $24: 871-873$.
9. FERRANDO (R.), LALLOUETTE (P.), BOURDERON (G.), FROGET(J.).- Influence d'un facteur de croissance du colibacille extrait d'Aspergillus flavus sur le gain de poids ef Ia flore intestinale. Comptes rendus AC. Sciences, 1956, No 5, 537.

10. FONTAINE (M.). - Pneumonie à virus du porc ou pneumonie enzootique. Généralités. Rec. Méd. Vét., 1964, 140, 1019-1025.

11. FOURNARAKI (A.) et ZERT (P.). - Le logement du porc. Edité par l'Institut technique du porc, 60, rue Caumartin, Paris IXe.

12. GORDON (W. A. M.), LUKE (D.), - Observations on restricted ventilation in pig houses. Vet. Rec., 1956, 68, 1030-1031.

13. GORET (P.), FONTAINE (M.), Mme FONTAINE (M. P.), - Recherches et considérations sur la pneumonie enzootique du porc. Rec. Méd. Vét., 1964, $140: 811-833$.

14. GORET (P.), JOUBERT (L.). - La désinfection continue en médecine vétérinaire. Nécessité d'une normalisation et d'un contrôle. Bull. Acad. Vét., 1960, 33, 329-333.

15. JEAN-BLAIN (M.), JOUBERT (L.), RUCKBUSCH (Y.), OUDAR (J.). - Vitamine A et infection pasteurellique expérimentale du porc. Sur l'étiologie de la « toux de porcherie ». Bull. Acad. Vét., 1960, 33, 275-285. 
16. JEAN-BLAIN (M.). - Etiologie ef pathogénie des maladies d'origine alimentaire. Rev. Méd. Vét., 1966, 117, 12, 1037-1057.

17. JOUBERT (L.). - La désinfection continue en médecine vétérinaire. Cahiers Méd. Vét., $1960,29, N^{\circ} 2,33-51$.

18. JOUBERT (L.). - Les maladies du porc ont changé de visage. Rev. Méd. Vét., 1959, 110 : 41-73.

19. MACKENZIE (A.). - Experimental observations on hungroorn infection together with virus pneumonia in pigs. Vet. Rec., $1963,75,114$.

20. PLACIDI (L.), HAAG (J.), - La pneumonie à virus du porc. Rec. Méd. Vét., 1956, 132, 5-20.

21. PETIT (J. P.), RIVIÉRE (R.), PERREAU (P.), PAGOT (J.). - Recherches sur l'Aflatoxine. Revue des travaux effectués pendant le 1 er semestre 1964 dans les laboratoires centraux de I'I. E. M. V. T. Rev, Elev, Méd. Vét. Pays Trop., 1964, 17, 239-253.

22. QUINCHON (C.). - Pneumonie enzootique du porc. Etapes du diagnostic ef du traitement. Rec. Méd. Vét., 1964, 140, 1027-1036.

23. RAYNAUD (J. P.). - Une dystrophie hépatique toxique du porc à Madagascar. Rev. Elev. Méd. Vét. Pays Trop., 1963, 16 (1), 23-32.

24. RENAULT (L.), VALLÉE (A.), QUINCHON (C.). - - Principaux sérotypes d'Escherichia Coli isolés chez le porc en France. Bull. Acod. Vét., 1965, 38, 465-476.

25. SERRES (H.), RAYNAUD (J. P.), THEODOSIADIS (G.), DAUMAS (R.), BOUR-
DIN (P.), RIBOT (J. J.). - La toxicité de certains tourteaux d'arachide à Madagascar. Bulletin de Madagascar, 1963, No 206, 575-580.

26. SERRES (H.). - La polyomyélite du pore à Madagascar (Revue des travaux effectués jusqu'en 1965). Revue de Médecine et d'Hygiène d'Outre-Mer, 1965, 343, 116-120.

27. SERRES ( $H$. ), ZEBROWSKA (N.), SEJOURNE (G.). - Note sur la complémentation du maïs ef du manios pour l'alimentation des porcs. Note technique de la Région de Recherches de Madagascar, 1966.

28. STEFANSKI (W.), - Rôles des parasites dans la transmission des maladies infectieuses du porc. Rec. Méd. Vét. 1956, 132, 585593.

29. THEODOSIADIS (G.). - Le caneton, réactif biologique pour le dépistage de la toxicité des tourteaux d'arachide. Rev. Elev. Méd. Vét. Poys Trop., 1963, 16, 229-236.

30. UNDERDAHL (N.R.), KELLEY (G. W.). L'exacerbation du pouvoir pathogène du virus de la pneumonie des porcs par la migration de larves d'Ascaris. J. Amer. Veter. Méd. Assoc., 1957, 130-173-175.

31. VAN GOIDSENHOVEN (Ch.), SCHOENAERS (F.). - Maladies infectieuses des animaux domestiques. Vigot Frères, Editeurs, Paris.

32. ZELENSKII (V.'P.). - Treatment of pasteurellosis in pigs. Sborn Trud. Leningrad manchno issled. Vet. Inst, 1963, 10, 215-228 (in Vet. Bull., 1964, 34, 6, No 2017-2018). 